\title{
AN INVESTIGATION OF THE MECHANISM OF THE Eu(III)/Eu(II) ELECTRODE REACTION IN THE PRESENCE OF EDTA
}

\author{
L. KISOVA*, M. SLUYTERS-REHBACH and J. H. SLUYTERS \\ Laboratory of Analytical Chemistry, State University, Utrecht (The Netherlands) \\ (Received 19th January 1972; in revised form 15th May 1972)
}

\section{INTRODUCTION}

In kinetic studies of electrode reactions, there seems to be a renewed interest in redox systems with both components soluble in the electrolyte solution. Since earlier studies of the influence of the concentration of the supporting electrolyte ${ }^{1}$, attention is currently directed to the effect of the nature of the supporting electrolyte, especially of its anion ${ }^{2-4}$. As was the case with the former type of studies ${ }^{1}$, phenomena connected with the latter ${ }^{2-4}$ appear to be associated with the well-known Frumkin effect $^{5}$. For example, the apparent rate constants of the $\mathrm{Cr}$ (III)/ $\mathrm{Cr}$ (II) couple in various electrolytes were different, but after applying the Frumkin correction the resulting true rate constants are very much alike ${ }^{2,4}$. A similar result has been obtained in our laboratory for the $\mathrm{Eu}(\mathrm{III}) / \mathrm{Eu}(\mathrm{II})$ couple in $1 \mathrm{M} \mathrm{KCl}$ and $\mathrm{KI}$, provided that for the Frumkin correction the proper $\phi_{2}$ potentials, -i.e. those calculated taking into account the presence of $\mathrm{Eu}(\mathrm{III})$ and $\mathrm{Eu}(\mathrm{II})$-were used ${ }^{3}$. However, a study ${ }^{4}$ of the same couple in $1 M \mathrm{KSCN}$ solution yielded a true rate constant which is substantially higher than that in $\mathrm{KCl}$ and $\mathrm{KI}$. Two alternative explanations were proposed, one concerned with the distance between the reaction plane and the Outer Helmholtz Plane and the other with the possibility that the reaction in thiocyanate proceeds via europium-SCN ${ }^{-}$complexes, which are present in significant amounts.

An argument supporting the latter explanation is the evidence in the literature $^{6-8}$, that the reduction of Eu(III) to $\mathrm{Eu}$ (II) becomes reversible in the presence of EDTA, which is known to form rather stable complexes with Eu(III) and Eu(II). As a reaction proceeding via complex species usually has interesting aspects this paper presents a more quantitative study of the europium reaction in the presence of EDTA. One point of particular interest is the question as to whether the complexes present in the solution dissociate or associate prior to the final charge transfer reaction. It is well-known that information on this problem can be gained by inspection of the exchange current density at the equilibrium potential as a function of ligand concentration ${ }^{9}$. Theories for this are available, either in a general form or specifically for amalgam-forming reactions ${ }^{9,10}$. Moreover as we prefer to determine the apparent rate constant instead of the exchange current density, it is necessary to reconsider the theory of the current-voltage equation for this special case. Our theory will be an extension of a treatment recently presented by De Kreuk et $a l^{4}$ for a simpler case.

\footnotetext{
* On leave from Department of Theoretical and Physical Chemistry, Purkyn University, Brno, Czechoslovakia.
} 


\section{THEORY}

\section{(i) The overall reaction}

In general, both components $\mathrm{O}$ and $\mathrm{R}$ of a redox couple may form various complexes with the ligand $\mathrm{L}$, which is assumed to be present in excess. The equilibrium potential $E_{\text {eq }}$ of an electrode immersed in a solution of $\mathrm{O}, \mathrm{R}, \mathrm{L}$ and the resulting complexes, can be written in various ways:

$$
\begin{aligned}
& E_{\text {eq }}=E^{0}+(R T / n F) \ln \left(c_{\mathrm{O}}^{*} / c_{\mathrm{R}}^{*}\right) \\
& E_{\text {eq }}=E_{p q}^{o}+(R T / n F) \ln \left(c_{\mathrm{OL}_{p}}^{*} / c_{\mathrm{RLq}}^{*}\right)
\end{aligned}
$$

or, quite operationally,

$$
E_{\text {eq }}=E_{\mathrm{T}}^{0}+(R T / n F) \ln \left(c_{\mathrm{OT}}^{*} / c_{\mathrm{RT}}^{*}\right)
$$

where $c_{\mathrm{O}}^{*}$ and $c_{\mathrm{R}}^{*}$ are the concentrations of the uncomplexed (aquo)-ions, $c_{\mathrm{OL}_{\mathrm{p}}}$ and $c_{\mathrm{RL}_{q}}^{*}$ the concentrations of the complexes $\mathrm{OL}_{p}$ and $\mathrm{RL}_{q}$ respectively, while $c_{\mathrm{OT}}^{*}$ and $c_{\mathrm{RT}}^{*}$ denote the analytical concentrations:

$$
\begin{aligned}
c_{\mathrm{OT}}^{*} & =c_{\mathrm{O}}^{*}+c_{\mathrm{OL}}^{*}+c_{\mathrm{OL}}^{*}+\ldots \\
& =c_{\mathrm{O}}^{*}\left[1+\beta_{\mathrm{O} 1} c_{\mathrm{L}}^{*}+\beta_{\mathrm{O} 2}\left(c_{\mathrm{L}}^{*}\right)^{2}+\ldots\right]=c_{\mathrm{O}}^{*} r_{\mathrm{O}} \\
c_{\mathrm{RT}}^{*} & =c_{\mathrm{R}}^{*}+c_{\mathrm{RL}}^{*}+c_{\mathrm{RL}}^{*}+\ldots \\
& =c_{\mathrm{R}}^{*}\left[1+\beta_{\mathrm{R} 1} c_{\mathrm{L}}^{*}+\beta_{\mathrm{R} 2}\left(c_{\mathrm{L}}^{*}\right)^{2}+\ldots\right]=c_{\mathrm{R}}^{*} r_{\mathrm{R}}
\end{aligned}
$$

If all the complexes of one species are in mobile equilibrium with each other, the limiting current $i_{1,0}$ of for example, the d.c. polarographic reduction wave is proportional to $c_{\mathrm{OT}}^{*} D_{\mathrm{O}}^{\frac{1}{2}}$, where $D_{\mathrm{O}}$ is the mean diffusion coefficient ${ }^{11}$. If the wave is reversible the half-wave potential is given by ${ }^{11-13}$

$$
\begin{aligned}
E_{\frac{1}{2}}^{\mathrm{r}} & =E^{0}+(R T / n F) \ln \left(D_{\mathrm{R}}^{\frac{1}{2}} / D_{\mathrm{O}}^{\frac{1}{3}}\right)+(R T / n F) \ln \left(r_{\mathrm{O}} / r_{\mathrm{R}}\right) \\
& =E_{\mathrm{T}}^{\mathbf{0}}+(R T / n F) \ln \left(D_{\mathrm{R}}^{\frac{1}{2}} / D_{\mathrm{O}}^{\frac{1}{2}}\right)
\end{aligned}
$$

According to eqn. (6) the half-wave potential is a complex function of $c_{\mathrm{L}}^{*}$, owing to the term in $r_{\mathrm{O}} / r_{\mathrm{R}}$. In the particular case that in both eqns. (4) and (5) one term, $c_{\mathrm{OL}_{p}}^{*}$ respectively $c_{\mathrm{RL}_{q}}^{*}$, dominates it is easily derived that:

$$
\begin{aligned}
E_{\frac{1}{2}}^{\mathrm{r}} & =E^{0}+(R T / n F) \ln \left(\beta_{\mathrm{R}_{q}} / \beta_{\mathrm{O}_{p}}\right)-(R T / n F) \ln \left(c_{\mathrm{L}}^{*}\right)^{p-q}+(R T / n F) \ln \left(D_{\mathrm{R}}^{\frac{1}{2}} / D_{\mathbf{O}}^{\frac{1}{2}}\right) \\
& =E_{p q}^{0}+(R T / n F) \ln \left(D_{\mathrm{R}}^{\frac{1}{2}} / D_{\mathrm{O}}^{\frac{1}{2}}\right)
\end{aligned}
$$

where $\beta_{\mathrm{O}_{p}}$ and $\beta_{\mathrm{R}_{q}}$ are the stability constants of the dominating complexes. In this case the integer $p-q$ can be derived from a plot of $E_{\frac{1}{2}} v s$. $\ln c_{\mathbf{L}}^{*}$.

\section{(ii) The charge transfer reaction}

In general, at the electrode surface every Ox complex may be reduced to a certain Red complex and vice versa. A theory based on this concept would be very complicated, since the current must be considered as the sum of a number of partial currents each pertaining to the reaction between a particular Ox- and Red-species. However, it may be conceivable that one of these partial reactions proceeds much faster than the others, either because of a low activation energy or because the reacting species is present in relatively high concentrations. In this case all the other 
complexes will dissociate or associate to form the species which is removed by the reaction, so that the overall reaction proceeds via these species. Evidence for the possibility of such a situation has been provided by Gerischer for a number of complexed metal ion-metal amalgam reactions ${ }^{10}$.

Let the intermediate charge transfer step be the reaction:

$$
\mathrm{OL}_{x}+n e \rightleftarrows \mathrm{RL}_{y}+(x-y) \mathrm{L}
$$

in which $x \geqslant y$.

The current-voltage characteristic for this reaction may be written as:

$$
i=n F k_{\text {sh }}^{i} \mathrm{e}^{\left(\beta n-z_{\mathrm{O}}\right) f \phi_{2}}\left\{\bar{c}_{\mathrm{RL}_{y}} \bar{c}_{\mathrm{L}}^{x-y} \mathrm{e}^{\alpha n f\left(E-E_{x y \mathrm{~L}}^{0}\right)}-\bar{c}_{\mathrm{OL}_{x}} \mathrm{e}^{-\beta n f\left(E-E_{x y \mathrm{~L}}^{0}\right)}\right\}
$$

in which $f=F / R T, z_{\mathrm{O}}$ is the charge of $\mathrm{OL}_{x}$ and $\phi_{2}$ is the potential in the reaction plane; $k_{\mathrm{sh}}$ is the rate constant, defined in such a way that it contains no concentration terms. Therefore $E_{x y \mathrm{~L}}^{0}$ is the standard potential for reaction (8), i.e.

$$
E_{\mathrm{eq}}=E_{x y \mathrm{~L}}^{0}+(R T / n F) \ln \left(c_{\mathrm{OL}_{x}}^{*} / c_{\mathrm{RL}_{y}}^{*}\left(c_{\mathrm{L}}^{*}\right)^{x-y}\right)
$$

As the stability constants of $\mathrm{OL}_{x}$ and $\mathrm{RL}_{y}$ will usually be unknown, $E_{x y \mathrm{~L}}^{0}$, as well as $\bar{c}_{\mathrm{RL}_{y}}$ and $\bar{c}_{\mathrm{OL}_{x}}$ will be experimentally inaccessible, Therefore we rearrange eqn. (9) with the aid of eqns. (3) and (10) to:

$$
\begin{aligned}
i= & n F k_{\mathrm{sh}} \mathrm{e}^{\left(\beta n-z_{\mathrm{O}}\right) f \phi_{2}}\left\{\frac{\bar{c}_{\mathrm{RL}_{y}}}{\bar{c}_{\mathrm{RT}}} \bar{c}_{\mathrm{L}}^{x-y}\left(\frac{c_{\mathrm{OL}}^{*} c_{\mathrm{RT}}^{*}}{c_{\mathrm{OT}}^{*} c_{\mathrm{R} \mathrm{L}_{y}}^{*}}\right)^{\alpha}\left(c_{\mathrm{L}}^{*}\right)^{\alpha(y-x)} \bar{c}_{\mathrm{RT}} \mathrm{e}^{a n f\left(E-E_{\mathrm{T}}^{0}\right)}\right. \\
& \left.-\frac{\bar{c}_{\mathrm{OL}_{x}}}{\bar{c}_{\mathrm{OT}}}\left(\frac{c_{\mathrm{OT}}^{*} c_{\mathrm{RL}}^{*}}{c_{\mathrm{OL}}^{*} c_{\mathrm{RT}}^{*}}\right)^{\beta}\left(c_{\mathrm{L}}^{*}\right)^{\beta(x-y)} \bar{c}_{\mathrm{OT}} \mathrm{e}^{-\beta n f\left(E-E_{\mathrm{T}}^{0}\right)}\right\}
\end{aligned}
$$

If the ligand is present in excess, we can put $\bar{c}_{\mathrm{L}}=c_{\mathrm{L}}^{*}$ and write further:

$$
\begin{aligned}
& \frac{\bar{c}_{\mathrm{OL}_{x}}}{\bar{c}_{\mathrm{OT}}}=\frac{c_{\mathrm{OL}}^{*}}{c_{\mathrm{OT}}^{*}}=\frac{c_{\mathrm{OL}}^{*} c_{\mathrm{O}}^{*}}{c_{\mathrm{O}}^{*} c_{\mathrm{OT}}^{*}}=r_{\mathrm{O}} \beta_{\mathrm{O}_{x}}\left(c_{\mathrm{L}}^{*}\right)^{x} \\
& \frac{\bar{c}_{\mathrm{RL}}}{\bar{c}_{\mathrm{RT}}}=\frac{c_{\mathrm{R} \mathrm{L}_{y}}^{*}}{c_{\mathrm{RT}}^{*}}=\frac{c_{\mathrm{R} \mathrm{L}_{y}}^{*} c_{\mathrm{R}}^{*}}{c_{\mathrm{R}}^{*} c_{\mathrm{RT}}^{*}}=r_{\mathrm{R}} \beta_{\mathrm{R}_{y}}\left(c_{\mathrm{L}}^{*}\right)^{y}
\end{aligned}
$$

where $\beta_{\mathrm{O}_{x}}$ and $\beta_{\mathrm{R}_{y}}$ are the stability constants of the reacting complexes.

Equation (11) can now be written as:

$$
i=n F k_{\mathrm{sh}}^{\mathbf{a}}\left\{\bar{c}_{\mathrm{RT}} \mathrm{e}^{\alpha n f\left(E-E \mathbf{E}_{\mathrm{T}}^{\mathbf{0}}\right)}-\bar{c}_{\mathrm{OT}} \mathrm{e}^{-\beta n F\left(E-E_{\mathrm{T}}^{0}\right)}\right\}
$$

in which the apparent rate constant is given by:

$$
k_{\mathrm{sh}}^{\mathrm{a}}=k_{\mathrm{sh}} \mathrm{e}^{\left(\beta n-z_{\mathrm{O}}\right) f \phi_{2}} \beta_{\mathrm{O}_{x}}^{\alpha} \beta_{\mathrm{R}_{y}}^{\beta} r_{\mathrm{O}}^{\alpha} r_{\mathrm{R}}^{\beta}\left(c_{\mathrm{L}}^{*}\right)^{x}
$$

Evidently $k_{\mathrm{sh}}^{\mathrm{a}}$ is a function of $c_{\mathrm{L}}^{*}$, in general in a complex manner because $r_{\mathrm{O}}$ and $r_{\mathrm{R}}$, defined by eqns. (4) and (5) are complex functions of $c_{\mathrm{L}}^{*}$. It may be noted that, if either $r_{\mathrm{O}}$ or $r_{\mathrm{R}}$ is known, the other of the two can be derived from $E_{\mathrm{T}}^{0}-E^{0}$, according to eqn. (6). In this case it is possible to determine $x$.

Again, if $\mathrm{Ox}$ and $\mathrm{Red}$ are present as dominating complexes $\mathrm{OL}_{p}$ and $\mathrm{RL}_{q}$, $r_{\mathrm{O}}$ and $r_{\mathrm{R}}$ can be expressed in terms of the stability constants of these complexes and we obtain :

$$
k_{\mathrm{sh}}^{\mathrm{a}}=k_{\mathrm{sh}} \mathrm{e}^{\left(\beta n-z_{\mathrm{o}}\right) f \phi_{2}}\left(\beta_{\mathrm{O}_{x}} / \beta_{\mathrm{O}_{p}}\right)^{\alpha}\left(\beta_{\mathrm{R}_{y}} / \beta_{\mathrm{R}_{q}}\right)^{\beta}\left(c_{\mathrm{L}}^{*}\right)^{x-\alpha p-\beta q} \text { for } x \geqslant y .
$$


Since in this case $p-q$ can be derived from the half-wave potential as a function of $c_{\mathrm{L}}^{*}$ (see eqn. (7), either $x-q$ or $x-p$ can be determined from $k_{\mathrm{sh}}^{\mathrm{a}}$ and $\alpha$. Note that even if the reaction proceeds via the dominating species $(x=p), k_{\mathrm{sh}}^{\mathrm{a}}$ still depends on $c_{\mathrm{L}}^{*}$ when $p \neq q$.

According to eqns. (14) the relation between $k_{\mathbf{s h}}^{\mathrm{a}}$ and $c_{\mathrm{L}}^{*}$ does not provide information on $y$, except that reaction (8) implies that $y \leqslant x$. If $y>x$, the reaction should be represented by:

$$
\mathrm{OL}_{x}+(y-x) \mathrm{L}+n e \rightleftarrows \mathrm{RL}_{y}
$$

and the current-voltage equation reads:

$$
i=n F k_{\mathrm{sh}} \mathrm{e}^{-\left(\alpha n+z_{\mathrm{R}}\right) f \phi_{2}}\left\{\bar{c}_{\mathrm{RL}} \mathrm{e}^{\alpha n F\left(E-E_{x y \mathrm{~L}}^{0}\right)}-\bar{c}_{\mathrm{OL}_{x}} \bar{c}_{\mathrm{L}}^{y-x} \mathrm{e}^{-\beta n f\left(E-E_{x y \mathrm{~L}}^{0}\right)}\right\}
$$

The term between brackets in eqn. (16) equals $\bar{c}_{\mathrm{x}}^{y-x}$ times the term between brackets in eqn. (9). Consequently the expression for $k_{\mathrm{sh}}^{\mathrm{a}}$ will be, by analogy with eqn. (14a)

$$
k_{\mathrm{sh}}^{a}=k_{\mathrm{sh}} \mathrm{e}^{-\left(\alpha n+z_{\mathrm{R}}\right) f \phi_{2}} \beta_{\mathrm{O}_{x}}^{\alpha} \beta_{\mathrm{R}_{y}}^{\beta} r_{\mathrm{O}}^{\alpha} r_{\mathrm{R}}^{\beta}\left(c_{\mathrm{L}}^{*}\right)^{y}
$$

Or, if the complexes $\mathrm{OL}_{p}$ and $\mathrm{RL}_{q}$ dominate in the solution, by analogy with eqn. $(14 b)$ :

$$
k_{\mathrm{sh}}^{a}=k_{\mathrm{sh}} \mathrm{e}^{-\left(\alpha n+z_{\mathrm{R}}\right) f \phi_{2}}\left(\beta_{\mathrm{O}_{x}} / \beta_{\mathrm{O}_{p}}\right)^{\alpha}\left(\beta_{\mathrm{R}_{\mathbf{y}}} / \beta_{\mathbf{R}_{q}}\right)^{\beta}\left(c_{\mathrm{L}}^{*}\right)^{y-\alpha p-\beta q}
$$

\section{EXPERIMENTAL}

Solutions were prepared by dissolving the required amounts of $\mathrm{Eu}_{2} \mathrm{O}_{3}$ in $\mathrm{HCl}$, adding EDTA in the form of $\mathrm{Na}_{2} \mathrm{H}_{2} \mathrm{Y}$ (where $\mathrm{Y}^{4-}$ represents the EDTA anion) and $\mathrm{NaOH}$ to bring the solution to $\mathrm{pH}$ 9.5. As a supporting electrolyte $\mathrm{NaCl}$ was added to a total ionic strength of $1 M$. All chemicals were reagent grade or p.a.

Measurements were made at $25^{\circ}$ in a three-electrode cell, with a dropping mercury electrode (DME), a mercury pool counterelectrode and a saturated calomel reference electrode (SCE). The complex impedance of this cell was measured as a function of frequency and of d.c. potential using the a.c. bridge described earlier ${ }^{14}$. D.c. polarograms were recorded using a Metrohm-Polarecord.

\section{RESULTS}

With regard to the composition of the solution several requirements had to be considered:

(i) In the theory it is assumed that EDTA is present in excess with regard to europium.

(ii) Besides EDTA it is preferable to have an indifferent electrolyte present in order to keep the ionic strength constant, e.g. at $1 M$. As EDTA was dissolved as the sodium salt, $\mathrm{NaCl}$ seemed useful for this purpose. The amount of EDTA should not be too large $(<0.1 M)$ in order to justify assumptions concerning the double layer, made in the Discussion section.

(iii) Rather high concentrations of $\mathrm{Eu}(\mathrm{III})(2$ or $5 \mathrm{mM})$ were necessary to obtain high accuracy in the impedance measurements.

(ii) and (iii) make it impossible to fulfil requirement (i) satisfactorily. Fortunately 
TABLE 1

ELECTROCHEMICAL PARAMETERS OF THE Eu(III)/Eu(II) REACTION IN MIXTURES OF $\mathrm{NaCl}$ AND $\mathrm{Na}_{2} \mathrm{H}_{2} \mathrm{Y}\left(\mathrm{Na}^{+}\right.$CONCENTRATION $\left.1 M\right)$ AT $\mathrm{pH} 9.5$

\begin{tabular}{|c|c|c|c|c|c|}
\hline $\begin{array}{l}\text { Analytical } \\
\text { Eu(III) concn. } \\
c_{O_{r}}^{*} / m M\end{array}$ & $\begin{array}{l}\text { Analytical } \\
\text { EDTA concn. } \\
c_{L_{T}}^{*} / m M\end{array}$ & $\begin{array}{l}c_{L T}^{*}-1.5 c_{O T}^{*} \\
=\bar{c}_{L}^{o} / m M\end{array}$ & $\begin{array}{c}-E_{\frac{1}{2}} / V \\
(v s . S C E)\end{array}$ & $\begin{array}{l}k_{\mathrm{s} h}^{a} / \\
c m s^{-1}\end{array}$ & $\alpha^{a}$ \\
\hline 5 & 100 & 92.5 & 1.18 & 0.016 & 0.62 \\
\hline 2 & 32 & 29 & 1.155 & 0.030 & 0.68 \\
\hline 5 & 15 & 7.5 & 1.115 & 0.076 & 0.58 \\
\hline 2 & 7 & 4 & 1.11 & 0.10 & 0.68 \\
\hline 5 & 10 & 2.5 & 1.09 & 0.17 & 0.25 \\
\hline
\end{tabular}

it appeared possible to drop this requirement. The theory supporting this is treated in the Appendix. Consequently, an analytical EDTA concentration $c_{\mathrm{LT}}^{*}$ between 7 and $100 \mathrm{mM}$ and analytical Eu(III) concentrations $c_{\text {Or }}^{*}$ of 2 or $5 \mathrm{mM}$ were chosen for the main series of experiments. The d.c. work was also done in a series of experiments with $c_{\mathrm{OT}}^{*}=0.2 \mathrm{mM}$.

The d.c. polarograms of all the solutions were reversible as appeared from the straight line with slope $1 / 0.059 \mathrm{~V}^{-1}$ obtained in plots of $\log \left[\left(i_{\mathrm{d}}-i\right) / i\right] v s$. $E$. The halfwave potentials obtained from these plots are tabulated in Table 1 . The mean diffusion coefficient, $D_{0}$, was found to be $(9 \pm 0.5) \times 10^{-6} \mathrm{~cm}^{2} \mathrm{~s}^{-1}$ in all solutions.

The impedance measurements were analysed in the usual way ${ }^{15}$, which implies that at each potential from the components $Z^{\prime}-R_{\Omega}$ and $Z^{\prime \prime}$ of the electrode impedance the components $Y_{\mathrm{el}}^{\prime}$ and $Y_{\mathrm{el}}^{\prime \prime}$ of the electrode admittance were calculated as a function of frequency. In order to diminish systematic errors due to "shielding" effects, $R_{\Omega}$ was taken as equal to the resistive component measured at each frequency at a potential outside the faradaic region ${ }^{16}$.

The frequency dispersion of $Y_{\mathrm{el}}^{\prime}$ and $Y_{\mathrm{el}}^{\prime \prime}$ could be fitted with sufficient precision to the parameters of the Randles circuit ${ }^{15}$, viz. the transfer resistance $\theta$, the Warburg coefficient $\sigma$ and the double layer capacity $C_{\mathrm{d}}$. As the electrode reaction appeared to be d.c. reversible, the parameters $\theta$ and $p^{\prime}=\theta / \sigma$ were inspected as a function of potential on the basis of the equations ${ }^{15}$ :

$$
\begin{aligned}
\theta & =\frac{R T\left(D_{\mathrm{R}} / D_{\mathrm{O}}\right)^{\frac{1}{2} \beta}}{n^{2} F^{2} k_{\mathrm{sh}}^{\mathrm{a}} c_{\mathrm{OT}}^{*}}\left\{\mathrm{e}^{\beta j}+\mathrm{e}^{-\alpha j}\right\} \\
p^{\prime} & =\frac{\left(2 D_{\mathrm{O}}^{\alpha} D_{\mathrm{R}}^{\beta}\right)^{\frac{1}{2}}}{k_{\mathrm{sh}}^{\mathrm{a}}} \frac{1}{\mathrm{e}^{-\beta j}+\mathrm{e}^{\alpha j}}
\end{aligned}
$$

where $j=n f\left(E-E_{\frac{1}{2}}\right)$, with $E_{\frac{1}{2}}$ corresponding to eqn. (10), i.e. the measured half-wave potential. The validity of these expressions follows immediately from application of the faradaic impedance theory to eqn. (13). Corresponding to the expressions for $\theta$ and $p^{\prime}$, the respective plots of $\log [\theta / 1+\exp (j)], \log \left[p^{\prime}(1+\exp (-j))\right]$ were straight lines, the slope of which yielded the apparent transfer coefficient $\alpha^{a}{ }^{\star}{ }^{*}$ For reasons given

\footnotetext{
* For the meaning of the concept "apparent transfer coefficient", see e.g. ref. 3.
} 
below, only the $\theta$ plots were used at the lower EDTA concentrations. From the interpolated value at $j=0, k_{\mathrm{sh}}^{\mathrm{a}}$ was calculated, with the assumption that $D_{\mathrm{R}}^{\frac{1}{2}}=D_{\bar{O}}^{\frac{1}{2}}=3 \times 10^{-3}$ $\mathrm{cm} \mathrm{s}^{-\frac{1}{2}}$. The results are given in Table 1 . For reasons given below the exceptional value $\alpha^{\mathrm{a}}=0.25$ is likely to be in error, so that for $\alpha^{\mathrm{a}}$ a mean value 0.64 can be adopted.

An experiment in the presence of $100 \mathrm{mM}$ EDTA was also done at $\mathrm{pH} 7$. The results were $k_{\mathrm{sh}}^{\mathrm{a}}=2 \times 10^{-2} \mathrm{~cm} \mathrm{~s}^{-1}, \alpha=0.68, E_{\frac{1}{2}}=-1.185 \mathrm{~V} v \mathrm{~s}$. SCE.

In order to get an idea of the influence of EDTA and its Eu complexes on the double layer structure, double layer capacities were determined in and outside the faradaic region, both in the presence and the absence of Eu(III). The essential results are represented in Figs. 1 and 2.
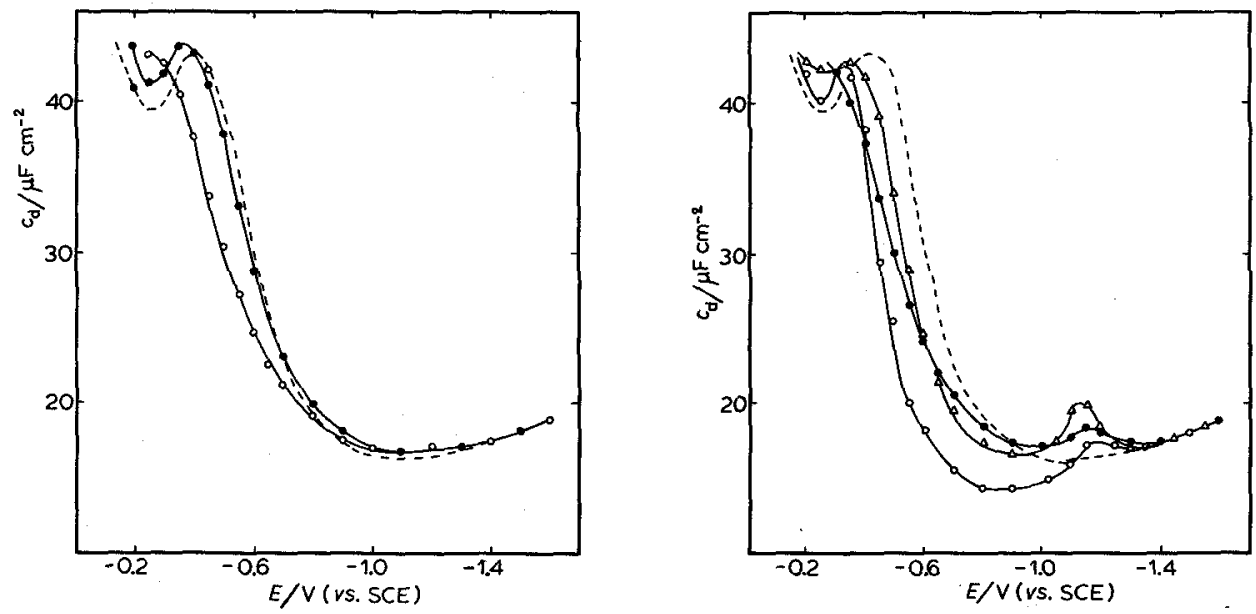

Fig. 1. Capacity-potential curves at pH 9.3 of the DME in solns. of: $(---) 0,(0) 10,(0) 100 \mathrm{mM}$ EDTA with $\mathrm{NaCl}$ added to an ionic strength of $1 M$.

Fig. 2. Capacity potential curves at pH 9.3 of the DME in solns. of $5 \mathrm{mM} \mathrm{Eu(III)} \mathrm{with} \mathrm{:} \mathrm{(O)} \mathrm{10,(} \triangle) 15$, (O) $100 \mathrm{~m} M$ EDTA. (--) $1 \mathrm{M}$ NaCl.

\section{DISCUSSION}

\section{(i) Neglecting double layer effects}

The half-wave potentials given in Table 1 are $c a .500 \mathrm{mV}$ more negative than the standard potential of the $\mathrm{Eu}(\mathrm{III}) / \mathrm{Eu}(\mathrm{II})$ couple in non-complexing medium, e.g. $-600 \mathrm{mV}$ in $1 \mathrm{M} \mathrm{NaClO}_{4}^{1}$. This means that $\mathrm{Eu}$ (III) must be more strongly complexed than $\mathrm{Eu}(\mathrm{II})$. Further, $E_{\frac{1}{2}}$ is a function of $c_{\mathrm{EDTA}}$ so that it is worthwhile to examine whether a relation of the type of eqn.(6) or(7) exists. In doing this, it must be recognized that the free EDTA concentration will differ considerably from the analytical concentration $c_{\mathrm{LT}}^{*}$, which for nearly all experiments does not sufficiently exceed the analytical $\mathrm{Eu}$ (III) concentration $c_{\mathrm{OT}}^{*}$. In the first instance this can be corrected for by assuming that the total amount of Eu(III) is complexed by at least one EDTA per ion. This is justified by the value of the half-wave potential, $500 \mathrm{mV}$ more negative than in perchlorate solutions. Thus, the maximum value of $\log r_{\mathrm{O}}=\log \left(c_{\mathrm{O}}^{*} / c_{\mathrm{OT}}^{*}\right)=-500 / 60=$ -8 . 
Figure 3 is a plot of $E_{\frac{1}{2}} v s . \log \left[c_{\mathrm{LT}}^{*}-c_{\mathrm{OT}}^{*}\right]$ i.e. the difference between the analytical concentrations of EDTA, both for the series with high Eu(III) concentration $(2-5 \mathrm{mM})$ and for the series with low $\mathrm{Eu}$ (III) concentration $(0.2 \mathrm{mM}$. Both plots are straight lines with slopes of $67 \mathrm{mV}$ and $60 \mathrm{mV}$, respectively. This indicates that in eqn. (7) $p-q \approx 1$. On the basis of the similarity in properties of $\mathrm{Eu}(\mathrm{II})$ and $\mathrm{Ba}(\mathrm{II})$, which has been pointed out by several authors ${ }^{7,8}$, it is reasonable to assume that $\mathrm{Eu}$ (II) is, like $\mathrm{Ba}(\mathrm{II})$, complexed by one ligand. This would mean that $q=1$ and $p=2$.

It is interesting to compare our result with the results of Onstott ${ }^{8}$, who carried out a similar study in different $\mathrm{pH}$ ranges. He found $p-q=1$ both for $6<\mathrm{pH}<8.5$ and $11<\mathrm{pH}<12$; however, in the intermediate region, $8.5<\mathrm{pH}<10.3$, his plot was irregular and certainly did not indicate that $p-q=1$, in contrast to our present result at $\mathrm{pH} 9.5$.

On the other hand, several other authors ${ }^{7,17,18}$ have studied the complexation of Eu(III) by EDTA and found reasons to assume that Eu(III) is attached to one EDTA ligand. As far as we can see, these authors do not give definite proof of this assumption. For example, Eckardt and Holleck ${ }^{7}$ assumed that the Eu(III)-EDTA complex would be similar to the Nd-EDTA complex, which was studied spectroscopically. Schwarzenbach and coworkers state only that they are dealing with 1:1 complexes. On the other hand we found a statement by Moeller and Ferrus ${ }^{19}$ that certain rare earth species have the ability to add more than one ligand, e.g. in the case of nitrilotriacetic acid.

An argument supporting $p=2$ can be given as follows. The solubility product of $\mathrm{Eu}(\mathrm{OH})_{3}$ is ${ }^{20}: 10^{-23}-3 \times 10^{-22}$. If according to Schwarzenbach, the complex EuY ${ }^{-}$exists with a stability constant of $5 \times 10^{16}$, an excess of only $10^{-8}-3 \times 10^{-7} \mathrm{mM}$ EDTA is required to prevent precipitation of $\mathrm{Eu}(\mathrm{OH})_{3}$ at $\mathrm{pH} 9.5$. However, when neutralizing an acid solution of $\mathrm{m} M \mathrm{Eu}$ (III) and $5 \mathrm{mM}$ EDTA to $\mathrm{pH} 9.5$, we observed a white precipitate which dissolved after addition of another $5 \mathrm{mM}$ EDTA. Evidently the ratio Eu(III):EDTA should be $1: 2$ in order to keep all the Eu(III) in solution.

It is not possible to decide from our experiments which EDTA species is the complexing ligand, since all species $\mathrm{H}_{4} \mathrm{Y}, \mathrm{H}_{3} \mathrm{Y}^{-}, \mathrm{H}_{2} \mathrm{Y}^{2-}, \mathrm{HY}^{3-}$ and $\mathrm{Y}^{4-}$ are in mobile equilibrium with each other. For the $\mathrm{Eu}$ (III) complex there is even the possibility of two different ligands, e.g. the complex $\mathrm{Eu}(\mathrm{HY}-\mathrm{Y})^{4-}$. In the following we will denote the complexing species by $\mathrm{L}$, without specifying its meaning. In the solutions with 2-5 $\mathrm{mM} \mathrm{Eu}$ (III), liberation of $\mathrm{L}$ during the electrode reaction might cause changes in $\mathrm{pH}$ near the electrode surface due to the fact that the EDTA is not always present in sufficient excess. This could lead to interference by complexes of the type Eu(Y$\mathrm{OH})$. According to the literature ${ }^{7,8}$, however, this occurs only at $\mathrm{pH}>12-12.8$. Unless $\mathrm{L}_{\mathrm{L}} \equiv \mathrm{Y}^{4-}$, the $\mathrm{pH}$ will become lower than 9.5 , owing to the presence of $\mathrm{Na}^{+}$ ions, which will cause reactions such as:

$$
\mathrm{HY}^{3-}+\mathrm{Na}^{+} \rightleftarrows \mathrm{NaY}^{3-}+\mathrm{H}^{+}
$$

It can be calculated that at $\mathrm{pH} 9.5,90 \%$ of EDTA not attached to europium is present as $\mathrm{NaY}^{3-}$ and $10 \%$ as $\mathrm{HY}^{3-}$. Therefore possible liberation of $\mathrm{Y}^{4-}$ from $5 \mathrm{mM} \mathrm{Eu}$ $\mathrm{Y}_{2}^{5-}$ will generate at the half-wave potential $0.25 \mathrm{mM} \mathrm{OH}{ }^{-}$ions, causing a shift to $\mathrm{pH}$ 10.4. This is well below the limit of $\mathrm{pH} 12$.

Another problem arises when EDTA is not present in excess. If $p-q=1$, we must conclude that it is not quite correct to plot $E_{\frac{1}{2}} v s . \log \left[c_{\mathrm{LT}}^{*}-c_{\mathrm{OT}}^{*}\right]$, as is done in 

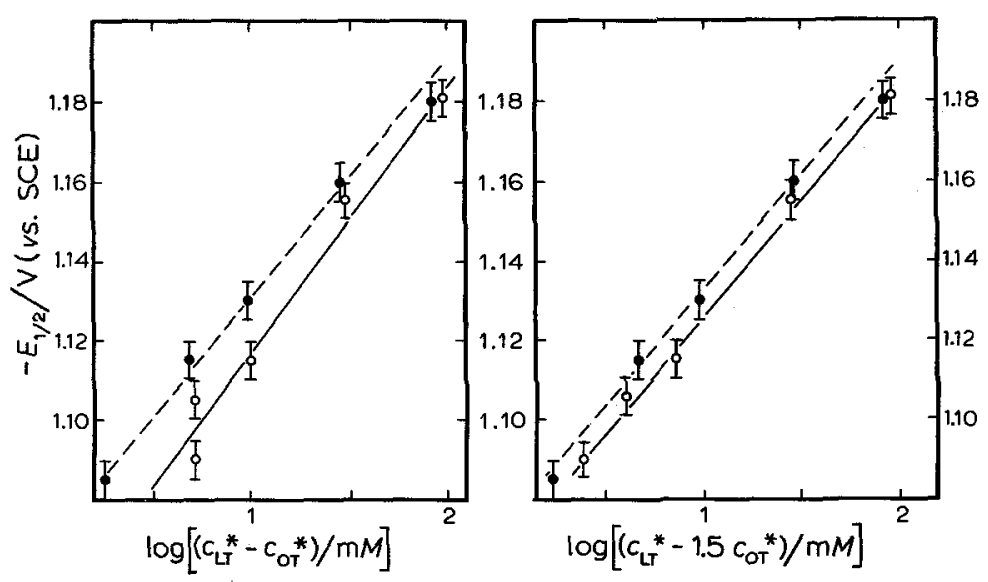

Fig. 3. Plot of half-wave potential $v s$. $\log$ of max. bulk concn. of free EDTA (see text). (O) 2 and $5 \mathrm{~m} M$ $\mathrm{Eu}(\mathrm{III}),(\mathrm{O}) 0.2 \mathrm{mM} \mathrm{Eu}(\mathrm{III})$.

Fig. 4. Plot of half-wave potential $v$ s. $\log$ of surface concn. of free EDTA at $E=E_{\frac{1}{2}}$, calcd. with $p=2$ and $q=1$ (see text). (O) 2 and $5 \mathrm{mM}$ Eu(III), (O) $0.2 \mathrm{mM} \mathrm{Eu(III).}$

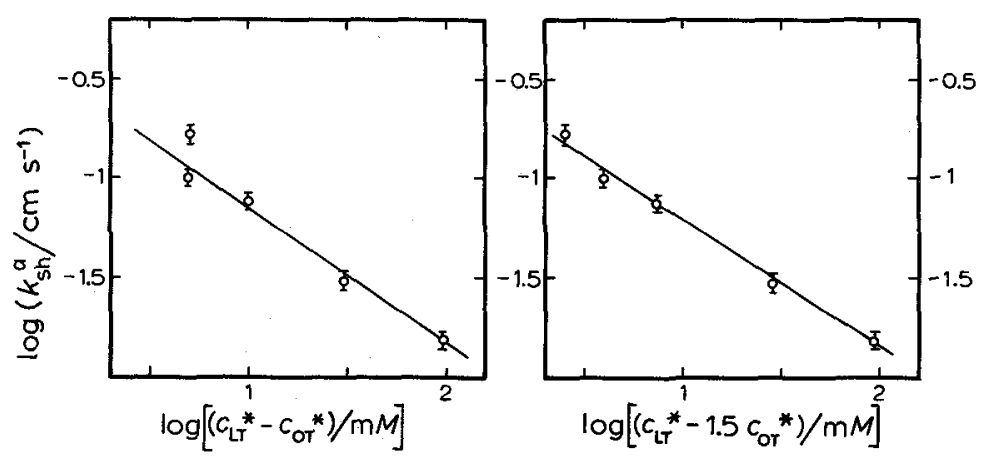

Fig. 5. Plot of $\log k_{\mathrm{sh}}^{\mathrm{a}} v \mathrm{~s}$. $\log$ of max. bulk concn. of free EDTA.

Fig. 6. Plot of $\log k_{\mathrm{sh}}^{\mathrm{a}} v$. $\log$ of surface concn. of free EDTA at $E=E_{\frac{1}{2}}$, calcd. with $p=2$ and $q=1$.

Fig. 3, since during the reduction of Eu(III), EDTA is liberated at the electrode surface. The consequences of this will be treated in an Appendix, where it will be shown that the correct variable to use for the abscissa is $c_{\mathrm{LT}}^{*}-\frac{1}{2}(p+q) c_{\mathrm{OT}}^{*}$. Actually this is the surface concentration at the half-wave potential, which we will denote by the symbol $\bar{c}_{\mathrm{L}}^{0}$. In Fig. 4 it is shown that this leads to a better fit; moreover, the slope of both new lines is $58 \mathrm{mV}$, so that the data are completely consistent with $p=2$ and $q=1$.

The fact that $c_{\mathrm{LT}}$ does not exceed $c_{\mathrm{OT}}^{*}$ sufficiently also leads to a problem if the effect of the ligand concentration on $k_{\mathrm{sh}}^{\mathrm{a}}$ is to be investigated. In the Appendix it will be derived that after replacement of $c_{\mathrm{L}}^{*}$ by $\bar{c}_{\mathrm{L}}^{\mathrm{O}}$, eqn. $(14 \mathrm{~b})$ or $(17 \mathrm{~b})$ remains applicable, provided that $k_{\mathrm{sh}}^{\mathrm{a}}$ is determined from the charge transfer resistance $\theta$ (and not from $p^{\prime}$ ).

In Figs. 5 and $6 \log k_{\mathrm{sh}}^{\mathrm{a}}$ is plotted $v s . \log \left(c_{\mathrm{LT}}^{*}-c_{\mathrm{OT}}^{*}\right)$ and $\log c_{\mathrm{LT}}^{*}-\left(\frac{1}{2} p+\frac{1}{2} q\right) c_{\mathrm{OT}}^{*}$. 
The slopes are -0.66 and -0.64 respectively, the latter, of course, being the more reliable. If eqn. (14b) holds, this means that $x=\alpha p+\beta q-0.64$, or with $\alpha$ equal to $\alpha^{\mathrm{a}}=$ $0.64 \pm 0.04, x=1.00 \pm 0.04$ and consequently $0 \leqslant y \leqslant 1$. If eqn. (17b) holds, we have $y=1.00 \pm 0.04$ and $0 \leqslant x \leqslant 1$. The most obvious conclusion is that the charge transfer reaction proceeds via the non-predominant species OL and the predominant species RL.

(ii) Possible influence of double layer effects

From the influence of EDTA on the capacity curves in Fig. 1 it can be concluded that it is adsorbed in the double layer. Although in our solutions the species $\mathrm{NaY}^{3-}$ is dominant, nothing can be said about the nature of the adsorbing species, since $\mathrm{H}_{4} \mathrm{Y}, \mathrm{H}_{3} \mathrm{Y}^{-}, \mathrm{H}_{2} \mathrm{Y}^{2}, \mathrm{HY}^{3-}$ and $\mathrm{Y}^{4-}$ and $\mathrm{NaY}^{3-}$ are in mobile equilibrium with each other. Since the Outer Helmholtz Plane potential $\phi_{2}$ is probably negative due to specific absorption of chloride ions, it is likely that the adsorption of EDTA is also specific.

The extent of the mutual differences between the curves in Fig. 1 sugggests that the adsorption is weak in the region of our interest, viz. -1.0 to $-1.25 \mathrm{~V} \mathrm{vs}$. SCE. From Fig. 2 it appears that the Eu(III)-EDTA complex is more strongly adsorbed, since at low free EDTA concentration the capacity is relatively more suppressed at potentials $>-0.8 \mathrm{~V}$. (at $0.1 \mathrm{M}$ EDTA concentration, addition of Eu(III) has no detectable influence). In the faradaic region small peaks are observed, also indicating adsorption of the electroactive species. According to recent developments ${ }^{21,22}$, the capacity values in these peak regions reflect the potential dependence of the electroactive and thus of the adsorbed species, rather than being a measure of the real double layer capacity, $\mathrm{d} q / \mathrm{d} E$. Although, in fact, the impedance analysis based on the classical Randles circuit is not correct in this case, the good fit we obtained and the smallness of the peaks suggest that the adsorption is very weak. Nevertheless, the influence of it on the double-layer structure may have a twofold effect on our results. First it is possible that adsorbed EDTA facilitates electron transfer to Eu-EDTA. However, one would expect in this case $k_{\mathbf{s h}}^{\mathrm{a}}$ to increase with EDTA concentration, whereas the opposite is found. Secondly, the OHP potential $\phi_{2}$ may be dependent on EDTA concentration and it is not a priori impossible that a proper correction for the Frumkin effect leads to a different value for $x$ or $y$. We will attempt to inspect this by speculation concerning a set of most probable cases, viz. $x=0, x=1$ and $x=2$ with either $\mathrm{H}_{2} \mathrm{Y}^{2-}, \mathrm{HY}^{3-}$ or $\mathrm{Y}^{4-}$ as the ligand, while $x \geqslant y$.

First, the true value of $\alpha$ should be calculated from $\alpha^{\mathrm{a}}$ for these cases, using the expression $^{3}$ :

$$
\alpha=\left[\alpha^{\mathrm{a}}-\left(1-\frac{z_{\mathrm{O}}}{n}\right) \frac{\mathrm{d} \phi_{2}}{\mathrm{~d} E}\right] /\left[1-\frac{\mathrm{d} \phi_{2}}{\mathrm{~d} E}\right]
$$

At potentials $\leqslant-1.0 \mathrm{~V}, \mathrm{~d} \phi_{2} / \mathrm{d} E=0.042$ for $1 \mathrm{M} \mathrm{NaCl}$; chloride ions are not adsorbed at these potentials ${ }^{23}$. If an EDTA anion is adsorbed, $\mathrm{d} \phi_{2} / \mathrm{d} E$ will be less, as can be inferred from a comparison of $\phi_{2}$ potentials in iodide ${ }^{24}$ and chloride ${ }^{23}$ solution at $E \leqslant-1.0 \mathrm{~V}$ vs. SCE. Therefore, taking $\mathrm{d} \phi_{2} / \mathrm{d} E$ as equal to 0.042 , we can calculate the extreme values of the true $\alpha$.

The value of the rate constant decreases by a factor 10 going from $\bar{c}_{\mathrm{L}}^{0}=2.5 \mathrm{mM}$ to $\bar{c}_{\mathrm{L}}^{0}=92.5 \mathrm{~m} M$. The ratio $(92.5 / 2.5)^{x-\alpha p-\beta q}$ can be calculated for each of the possible 


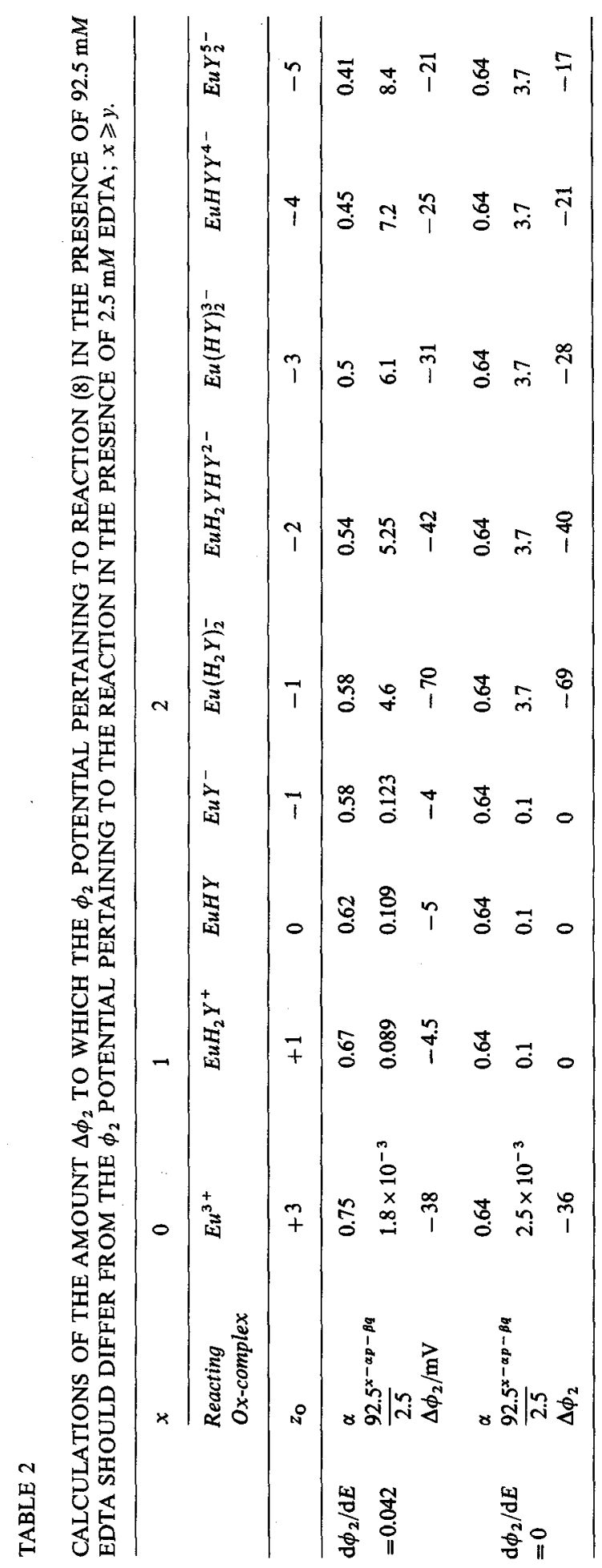

J. Electroanal. Chem., 40 (1972) 
reacting complexes, using the true value of $\alpha$ combined with $p=2$ and $q=1$ as the most probable bulk coordination numbers. It may be noted that the result $p-q=1$, obtained from Fig. 4, remains valid, since possible adsorption of reaction species and/or ligand has no influence on the validity of eqns. $(10)$ and $(11)^{25}$. When the calculated ratio differs from 0.1 , this can only be accounted for by a difference in $\phi_{2}$ pertaining to the $2.5 \mathrm{mM}$ and the $92.5 \mathrm{mM}$ solutions. The amount of this difference is calculated in accordance with eqn. (14b). Results are given in Table $2 . \mathrm{d} \phi_{2} / \mathrm{d} E=0$ as well as $\mathrm{d} \phi_{2} / \mathrm{d} E=$ 0.042 has been selected as a starting point in order to show that the value of this quantity does not influence the general trend.

The other set of possibilities, $y=0, y=1$ or $y=2$ with $\mathrm{H}_{2} \mathrm{Y}^{2-}, \mathrm{HY}^{3-}$ or $\mathrm{Y}^{4-}$ as the ligand, should be considered if $y>x$. The results are identical with those of Table 2 if $x$ is replaced by $y$ and the reacting Ox-complexes by the corresponding Redcomplexes. This is because for every column $z_{\mathrm{R}}=z_{\mathrm{O}}-1$, so that the Frumkin correction terms in eqns. (14b) and (17b) become identical $\left(-\alpha n-z_{\mathrm{R}}=\beta n-z_{\mathrm{O}}\right)$.

The results of Table 2 are noteworthy. Both the possibilities $x=0$ and $x=2$, and $y=0$ and $y=2$, require that $\phi_{2}$ at $\bar{c}_{\mathrm{L}}^{0}=92.5 \mathrm{mM}$ be at least $20 \mathrm{mV}$ more negative than $\phi_{2}$ pertaining to $\bar{c}_{\mathrm{L}}^{0}=2.5 \mathrm{mM}$. In view of the capacity curves in Figs. 1 and 2 it seems rather improbable that EDTA is adsorbed so strongly that such a large difference is real. For $x=1$, and $y=1$, the difference is -4 to $-5 \mathrm{mV}$, almost independent of the type of reacting complex. This is in excellent agreement with the potential dependence of $\phi_{2}$ in $1 \mathrm{M} \mathrm{NaCl}$ : at $E=-1.09 \mathrm{~V}$ ( $E_{\frac{1}{2}}$ pertaining to $2.5 \mathrm{mM}$ EDTA) $\phi_{2}=-49.5 \mathrm{mV}$ and at $E=-1.18 \mathrm{~V} v \mathrm{~s}$. $\operatorname{SCE}\left(E_{\frac{1}{2}}\right.$ found in $92.5 \mathrm{~m} M$ EDTA $\phi_{2}=-53.5 \mathrm{mV}$.

\section{CONCLUSIONS}

The data and analysis, presented in this paper, are consistent with the following conclusions : At pH 9.5 both Eu(III) and Eu(II) form selective complexes with EDTA with the respective coordination numbers 2 and 1 . The former coordination number is rather unusual and further studies at different $\mathrm{pH}$-values appear to be required.

The $\mathrm{Eu}(\mathrm{III}) / \mathrm{Eu}(\mathrm{II})$ reaction proceeds in the presence of EDTA following the mechanism:

$$
\begin{aligned}
& \mathrm{Eu}(\mathrm{III}) \mathrm{L}_{2} \rightleftarrows \mathrm{Eu}(\mathrm{III}) \mathrm{L}+\mathrm{L} \quad \text { (Fast association/dissociation) } \\
& \mathrm{Eu}(\mathrm{III}) \mathrm{L}+e \rightleftarrows \mathrm{Eu}(\mathrm{II}) \mathrm{L} \quad \text { (Charge transfer) }
\end{aligned}
$$

Both EDTA and Eu(III)(EDTA) ${ }_{2}$ are adsorbed at the mercury- $\mathrm{NaCl}$ solution interface. The adsorption is relatively weak and it is improbable that its influence on the double layer structure invalidates the mechanism proposed. However, it is worthwhile to check this more precisely on the basis of a detailed investigation of the double layer properties pertaining to the relevant solutions.

The values of the apparent rate constants may be compared qualitatively with the values found in other media, viz. $\mathrm{KCl}, \mathrm{KI}^{3}$ and $\mathrm{KSCN}^{4}$. The factors determining $k_{\mathrm{sh}}^{\mathrm{a}}$ according to eqn. (14b) can be estimated as follows:

With $\beta=1-\alpha=0.38$ (see Table 2), $z_{0}=0$ and $\phi_{2}=-49.5$ to $-53.5 \mathrm{mV}$ (taken from data for $1 M \mathrm{NaCl}), \exp \left[\left(\beta n-z_{\mathrm{O}}\right) f \phi_{2}\right]=0.5-0.45 . \beta_{\mathrm{Ox}} / \beta_{\mathrm{O} p} \leqslant 0.01$, so $\left(\beta_{\mathrm{O} x} / \beta_{\mathrm{O} p}\right)^{\alpha} \leqslant 0.05 . \beta_{\mathrm{R} y} / \beta_{\mathrm{R} q}=1$, since $y=q .\left(\bar{c}_{\mathrm{L}}^{0}\right)^{x-\alpha p-\beta q}$ ranges from 4.5 at $\bar{c}_{\mathrm{L}}^{0}=0.0925$ to 45 at $\bar{c}_{\mathrm{L}}{ }^{0}=0.025 \mathrm{M}$. This leads to $k_{\mathrm{sh}} \geqslant 0.16$. This value is considerably higher than 
the true constants in $\mathrm{KCl}\left(2 \times 10^{-5}\right), \mathrm{KI}\left(0.75 \times 10^{-5}\right)$ and $\mathrm{KSCN}\left(3.2 \times 10^{-4}\right)^{4}$. It can therefore be concluded that complexation by EDTA accelerates the charge transfer process of the $\mathrm{Eu}(\mathrm{III}) / \mathrm{Eu}(\mathrm{II})$ reaction.

\section{ACKNOWLEDGEMENT}

This investigation was supported in part by the Netherlands Foundation for Chemical Research (SON) with financial aid from the Netherlands Organisation for the Advancement of Pure Research (ZWO).

SUMMARY

An a.c. and d.c. polarographic study has been made of the $\mathrm{Eu}(\mathrm{III}) / \mathrm{Eu}(\mathrm{II})$ electrode reaction at the dropping mercury electrode in solutions containing different amounts of EDTA and $\mathrm{NaCl}$ (ionic strength $1 M, \mathrm{pH} 9.5$ ). Both $\mathrm{Eu}$ (III) and $\mathrm{Eu}$ (II) appear to be strongly complexed by EDTA with coordination numbers of two and one respectively, $A$ theory is developed which relates the rate constant of the charge transfer reaction to the free ligand concentration. Application of this relation to the rate constants and charge transfer coefficients obtained experimentally leads to the conclusion that both the Ox- and Red-component involved in the charge transfer reaction are Eu-EDTA complexes, with coordination number one.

APPENDIX

Implications of insufficient excess of ligand

(i) Premises. The aim of this Appendix is to justify the method applied in the analysis of the half-wave potential and the faradaic impedance for correcting for an insufficient excess of ligand. For the sake of simplicity, use will be made of some of the results, viz. the fact that the reaction is d.c. reversible and that in the bulk solution $\mathrm{Eu}(\mathrm{III})$ and $\mathrm{Eu}(\mathrm{II})$ form predominant complexes $\mathrm{OL}_{p}$ and $\mathrm{RL}_{q}$, the former being present originally, the latter generated by a d.c. current.

(ii) The half-wave potential. If $p \neq q$, reduction of $\mathrm{OL}_{p}$ to $\mathrm{RL}_{q}$ causes a change in the surface concentration $\bar{c}_{\mathrm{L}}$ of the ligand, since the overall reaction is:

$$
\mathrm{OL}_{p}+n e \rightleftarrows \mathrm{RL}_{q}+(p-q) \mathrm{L}
$$

Concentration gradients of $\mathrm{OL}_{p}, \mathrm{RL}_{q}$ and $\mathrm{L}$ will exist in the diffusion layer, according to:

$$
\frac{i}{n F}=\frac{D_{\delta}^{\frac{1}{3}}}{(h t)^{\frac{1}{2}}}\left(\bar{c}_{\mathrm{OL}_{p}}-c_{\mathrm{OL}_{p}}^{*}\right)=-\frac{D_{\mathrm{R}}^{\frac{1}{2}}}{(h t)^{\frac{1}{2}}} \bar{c}_{\mathrm{RL}_{q}}=-\frac{1}{p-q} \frac{D_{\mathrm{L}}^{\frac{1}{2}}}{(h t)^{\frac{1}{2}}}\left(\bar{c}_{\mathrm{L}}-c_{\mathrm{L}}^{*}\right)
$$

For a d.c. reversible reaction the Nernst equation may be applied to the surface concentrations:

$$
E=E^{0}+(R T / n F) \ln \left(\bar{c}_{\mathrm{O}} / \bar{c}_{\mathrm{R}}\right)=E_{p q}^{0}+(R T / n F) \ln \left(\bar{c}_{\mathrm{OL}_{p}} / \bar{c}_{\mathrm{RL}_{q}}\right)
$$

from which follows: 


$$
\begin{aligned}
E_{p q}^{0} & =E^{0}+(R T / n F) \ln \left(\bar{c}_{\mathrm{O}} \bar{c}_{\mathrm{RL}_{q}}\right) /\left(\bar{c}_{\mathrm{OL}_{p}} \bar{c}_{\mathrm{R}}\right) \\
& =E^{0}+(R T / n F) \ln \left(\beta_{\mathrm{R}_{q}} / \beta_{\mathrm{O}_{p}}\right)-(R T / n F) \ln \left(\bar{c}_{\mathrm{L}}\right)^{p-q}
\end{aligned}
$$

As $c_{\mathrm{RL}_{q}}^{*}=0$, the anodic limiting current is zero. For the cathodic limiting current there are two possibilities: $i_{\mathrm{I}}=-n F\left(D_{\mathrm{O}} / h t\right)^{\frac{1}{2}} c_{\mathrm{OL}}^{*}$ if $p-q>0$ or if $p-q<0$ but $c_{\mathrm{L}}^{*}>c_{\mathrm{OL}_{p}}^{*}$. If, however, $p-q<0$ and $c_{\mathrm{L}}^{*}<c_{\mathrm{OL}}^{*}$, we have $i_{l}=-n F\left(D_{\mathrm{L}} / h t\right)^{\frac{1}{2}} c_{\mathrm{L}}^{*} / q-p$. In the following it will be assumed that the conditions for the latter possibility are not fulfilled. Then at the half-wave potential, where $i=\frac{1}{2} i_{l}$, from eqn. (A2):

$$
\begin{aligned}
& \overline{\mathrm{OL}}_{p}=\frac{1}{2} c_{\mathrm{OL}_{p}}^{*}=\bar{c}_{\mathrm{RL}_{q}}\left(D_{\mathrm{R}} / D_{\mathrm{O}}\right)^{\frac{1}{2}} \\
& \bar{c}_{\mathrm{L}}=c_{\mathrm{L}}^{*}+\frac{1}{2}(p-q) c_{\mathrm{OL}_{p}}^{*}\left(D_{\mathrm{O}} / D_{\mathrm{L}}\right)^{\frac{1}{2}} \approx c_{\mathrm{LT}}^{*}-\frac{1}{2}(p+q) c_{\mathrm{OL}_{p}}^{*}
\end{aligned}
$$

in which $c_{\mathrm{LT}}^{*}$ is the analytical concentration of the ligand, being equal to $c_{\mathrm{L}}^{*}+p c_{\mathrm{O}_{p}}^{*}$. Neglect of the term $\left(D_{\mathrm{O}} / D_{\mathrm{L}}\right)^{\frac{1}{2}}$ will cause only a slight error, so that the approximation in (A6) can be combined with eqns. (A3)-(A5) to give for the half-wave potential:

$$
\begin{aligned}
& E_{\frac{1}{2}}=E^{0}+(R T / n F) \ln \left(\beta_{\mathrm{R}_{q}} / \beta_{\mathrm{O}_{n}}\right)+(R T / n F) \ln \left(D_{\mathrm{R}}^{\frac{1}{\mathrm{R}}} / D_{\mathrm{O}}^{\frac{1}{\mathrm{O}}}\right) \\
&-(R T / n F) \ln \left(c_{\mathrm{LT}_{\mathrm{T}}}^{*}-\frac{1}{2}(p+q) c_{\mathrm{OL}_{p}}^{*}\right)^{p-q}
\end{aligned}
$$

By selecting the proper values for $p$ and $q$ the most consistent fit can be found.

(iii) The faradaic impedance. The expressions (18) and (19) used for the parameters of the faradaic impedance are based on the analogy between eqn. (13) and the starting equation used in the general theory for small-amplitude a.c. perturbations published earlier ${ }^{15}$. If $\bar{c}_{\mathrm{L}}$ is to be considered as a variable, a similar derivation must be performed starting with eqn. (9), which can also be written as:

$$
\begin{array}{r}
i=n F k_{\mathrm{sh}} \mathrm{e}^{\left(\beta n-z_{\mathrm{O}}\right) f \phi_{2}} \bar{c}_{\mathrm{L}}^{x-p}\left\{\left(\beta_{\mathrm{R}_{y}} / \beta_{\mathrm{R}_{q}}\right) \bar{c}_{\mathrm{RL}_{q}} \bar{c}_{\mathrm{L}}^{p-q} \mathrm{e}^{\alpha n f\left(E-E_{x y \mathrm{~L}}^{\mathrm{o}}\right)}\right. \\
\left.-\left(\beta_{\mathrm{O}_{x}} / \beta_{\mathrm{O}_{p}}\right) \bar{c}_{\mathrm{OL}_{p}} \mathrm{e}^{-\beta n f\left(E-E_{x y \mathrm{~L}}^{\mathbf{0}}\right)}\right\}
\end{array}
$$

If the current consists of an a.c. current $\Delta i=i_{m} \sin \omega t$ added to a d.c. current $i$, eqn. (A8) becomes $^{15}$ :

$$
\begin{aligned}
\frac{i+\Delta i}{n F k_{\mathrm{sh}} \mathrm{e}^{\left(\beta n-z_{\mathrm{O}}\right) f \phi 2}}= & (1+g n f \Delta E)\left(\bar{c}_{\mathrm{L}}+\Delta c_{\mathrm{L}}\right)^{x-p} \\
& \times\left\{\frac{\beta_{\mathrm{R}_{y}}}{\beta_{\mathrm{R}_{q}}}\left(\bar{c}_{\mathrm{RL}_{q}}+\Delta c_{\mathrm{RL}_{q}}\right)\left(\bar{c}_{\mathrm{L}}+\Delta c_{\mathrm{L}}\right)^{p-q}(1+\alpha n F \Delta E) \mathrm{e}^{\alpha \varphi}\right. \\
& \left.-\frac{\beta_{\mathrm{O}_{x}}}{\beta_{\mathrm{O}_{p}}}\left(\bar{c}_{\mathrm{OL}_{p}}+\Delta c_{\mathrm{OL}_{p}}\right)(1-\beta n f \Delta E) \mathrm{e}^{-\beta \varphi}\right\}
\end{aligned}
$$

in which:

$$
\begin{aligned}
& g=\frac{1}{n f} \frac{\mathrm{d}\left(\ln k_{\mathrm{sh}}\right)}{d E}+\left(\beta-\frac{z_{\mathrm{O}}}{n}\right) \frac{\mathrm{d} \phi_{2}}{\mathrm{~d} E} \\
& \varphi=n f\left(E-E_{x y \mathrm{~L}}^{0}\right)
\end{aligned}
$$

The a.c. parts $\Delta c_{\mathrm{i}}$ of the concentration terms are related to $\Delta_{\mathrm{i}}$ by the Warburg equations $^{12}$. For $\Delta c_{\mathrm{RL}_{q}}$ and $\Delta c_{\mathrm{OL}_{p}} \neq 1$ we have: 


$$
\begin{aligned}
\Delta c_{\mathrm{OL}_{p}} & =\frac{i_{\mathrm{m}}}{n F\left(2 \omega D_{\mathrm{O}}\right)^{\frac{1}{2}}}(\sin \omega t-\cos \omega t) \\
\Delta c_{\mathrm{RL}_{q}} & =-\frac{i_{\mathrm{m}}}{n F\left(2 \omega D_{\mathrm{R}}\right)^{\frac{1}{2}}}(\sin \omega t-\cos \omega t)
\end{aligned}
$$

and for $\Delta c_{\mathrm{L}}$, by analogy:

$$
\Delta c_{\mathrm{L}}=-\frac{(p-q) i_{\mathrm{m}}}{n F\left(2 \omega D_{\mathrm{L}}\right)^{\frac{1}{2}}}(\sin \omega t-\cos \omega t)
$$

After selection of the first order a.c. terms from (A9) and introduction of eqns. (A12), $\Delta E$ can be expressed as a function of $\Delta i$ and thus the general expression for the faradaic impedance will be obtained. Before doing this, we introduce the simplifying Nernst equation which holds if the reaction is d.c. reversible. This implies that:

$$
\mathrm{e}^{\varphi}=\frac{\bar{c}_{\mathrm{OL}_{x}}}{\bar{c}_{\mathbf{R L}_{y}} \bar{c}_{\mathbf{L}}^{x-y}}=\frac{\bar{c}_{\mathrm{OL}_{p}}}{\bar{c}_{\mathbf{R L}_{q}}} \frac{\beta_{\mathrm{O}_{x}} \beta_{\mathrm{R}_{q}}}{\beta_{\mathrm{O}_{p}} \beta_{\mathbf{R}_{y}}} \bar{c}_{\mathrm{L}}^{(p-q)}
$$

which yields:

$$
\begin{aligned}
\frac{i_{\mathrm{m}} \sin \omega t}{n F k_{\mathrm{sh}} \mathrm{e}^{(\beta n-z \mathrm{O}) f \phi_{2}}}=\left(\frac{\beta_{\mathrm{O}_{x}}}{\beta_{\mathrm{O}_{p}}}\right)^{\alpha}\left(\frac{\beta_{\mathrm{R}_{\mathrm{y}}}}{\beta_{\mathrm{R}_{q}}}\right)^{\beta} \bar{c}_{\mathrm{L}}^{x-\alpha p-\beta q} \bar{c}_{\mathbf{O L}_{p}}^{\alpha} \bar{c}_{\mathrm{RL}_{q}}^{\beta} \\
\times\left\{n f \Delta E-\frac{\Delta c_{\mathrm{OL}_{p}}}{\bar{c}_{\mathrm{OL}_{p}}}+\frac{\Delta c_{\mathrm{RL}_{q}}}{\bar{c}_{\mathrm{RL}_{q}}}+(p-q) \frac{\Delta c_{\mathrm{L}}}{\bar{c}_{\mathrm{L}}}\right\}
\end{aligned}
$$

Consequently the relation between $\Delta E$ and $\Delta i$ can be written as:

$$
\Delta E=\theta i_{\mathrm{m}} \sin \omega t+\sigma \omega^{-\frac{1}{2}} i_{\mathrm{m}}(\sin \omega t-\cos \omega t)
$$

where $\theta$ follows immediately from eqn. (A14):

$$
\begin{aligned}
\theta= & \frac{R T}{n^{2} F^{2}} \\
& \times \frac{1}{k_{\mathrm{sh}} \mathrm{e}^{\left(\beta n-z_{\mathbf{O}}\right) f \phi_{2}}\left(\beta_{\mathrm{O}_{x}} / \beta_{\mathrm{O}_{p}}\right)^{\alpha}\left(\beta_{\mathrm{R}_{y}} / \beta_{\mathrm{R}_{q}}\right)^{\beta} \bar{c}_{\mathrm{L}}^{x-\alpha p-\beta q} \bar{c}_{\mathrm{OL}_{p}}^{\alpha} \overline{\mathrm{RL}}_{\mathrm{R} q}^{\beta}}
\end{aligned}
$$

\section{REFERENCES}

1 (a) L. Gierst in E. Yeager (Ed.), Transactions of the Symposium on Electrode Processes, Wiley, New York, 1961 , p. 109.

(b) L. Gierst and R. Cornelissen, Collect. Czech. Chem. Commun., 25 (1960) 3004.

2 F. C. Anson, N. Rathjen and R. D. Frisbee, J. Electrochem. Soc., 117 (1970) 477.

3 C. W. de Kreuk, M. Sluyters-Rehbach and J. H. Sluyters, J. Electroanal. Chem., 33 (1971) 267.

4 C. W. de Kreuk, M. Sluyters-Rehbach and J. H. Sluyters, J. Electroanal. Chem., 28 (1970) 391.

5 A. Frumkin, Z. Phys. Chem., 164A (1933) 121.

6 A. A. Vlček, Collect. Czech. Chem. Commun., 20 (1955) 1507; 24 (1959) 181.

7 D. Eckhardt and L. Holleck, Z. Elektrochem., 59 (1955) 202.

8 E. I. Onstott, J. Amer. Chem. Soc., 74 (1952) 3773.

9 H. Gerischer, Z. Phys. Chem. Leipz., 202 (1953) 292, 302.

10 P. Delahay, Double Layer and Electrode Kinetics, Wiley, New York, 1965, p. 183.

J. Electroanal. Chem., $40(1972)$ 
11 J. Heyrovský and J. Kůta, Principles of Polarography, Academic Press, New York, London, 1966. 12 J. J. Lingane, Chem. Rev., 29 (1941) 1.

13 D. R. Crow, Polarography of Metal Complexes, Academic Press, New York, London, 1966.

14 M. Sluyters-Rehbach and J. H. Sluyters, Recl. Trav. Chim., 82 (1963) 525.

15 M. Sluyters-Rehbach and J. H. Sluyters in A. J. Bard (Ed.), Electroanalytical Chemistry, Vol. 4, Marcel Dekker, New York, 1970.

16 C. W. de Kreuk, M. Sluyters-Rehbach and J. H. Sluyters, J. Electroanal. Chem., 35 (1972) 137.

17 G. Schwarzenbach, R. Gut and G. Anderegg, Helv. Chim. Acta, 37 (1954) 937.

18 E. J. Weelwright, F. H. Spedding and G. Schwarzenbach, J. Amer. Chem. Soc., 75 (1953) 4196.

19 T. Moeller and R. Ferrus, Inorg. Chem., 1 (1962) 49.

20 L. G. Sillen, Stability Constants of Metal-Ion Complexes, Metcalfe and Cooper, London, 1964.

21 P. Delahay, J. Phys. Chem., 70 (1966) 2067, 2373.

$22 \mathrm{R}$ Parsons in P. Delahay (Ed.), Advances in Electrochemistry and Electrochemical Engineering, Wiley, New York, 1970, p. 177.

23 D. C. Grahame and R. Parsons, J. Amer. Chem. Soc., 83 (1961) 1291.

24 D. C. Grahame, J. Amer. Chem. Soc., 80 (1958) 4201.

25 M. Sluyters-Rehbach and J. H. Sluyters, J. Electroanal. Chem., 39 (1972) 339.

J. Electroanal. Chem., 40 (1972) 